Paru comme chapitre 1, p17-27, in J. Lebeaume, A. Hasni, I. Harlé (dir.), Recherches et expertises pour l'enseignement scientifique. Bruxelles : De Boeck, 2011.

\title{
Relations entre recherche et expertise \\ dans un laboratoire de didactique curriculaire de sciences et techniques ${ }^{1}$
}

Jean-Louis Martinand, UMR_P1.STEF, INRP-ENS Cachan, Universud-Paris

$\underline{\text { martinan@stef.ens-cachan.fr }}$

Tous les laboratoires de recherche sur des pratiques sont confrontés à des demandes de conseil, d'expertise, d'intervention en formation, de production documentaire. Les laboratoires, les chercheurs en éducation, les didacticiens échappent d'autant moins à une demande externe et un besoin interne pour ces actions qui ne relèvent pas directement de la recherche, car à la différence des «sciences fondamentales », leurs disciplines peuvent difficilement se passer d'un tel investissement qui les justifie dans la société. En même temps, ces chercheurs et disciplines adoptent un positionnement ambivalent, se méfiant de l' «instrumentalisation » et visant la mise à jour de connaissances scientifiques pour certains, ou au contraire trouvant dans ces occasions de sortir du laboratoire à la fois une légitimation et des motifs à nouvelles recherches.

La question que ce texte veut poser est la suivante : avec quelles idées et quels « modèles » en tête abordons-nous ces fonctions à la fois proches et différentes de la recherche ? Disposons-

\footnotetext{
${ }^{1}$ Ce texte est une version remaniée d'une conférence «Entre pratique enseignante, formation et recherche : circulation des savoirs et reproblématisation » Fès (23-11-2006). Une présentation plus générale de ces questions a fait l'objet de la partie «entretien» d'un dossier de revue sur «Les savoirs entre pratique, formation et recherche » (Burguière \& Martinand, 2002).
} 
nous des bonnes « représentations » pour penser les tâches et les rôles de chercheur dans ces situations? Qu'avons-nous à changer?

1. Comme chercheurs, nous aimerions beaucoup croire à un schéma de transfert - application du savoir. La recherche serait le lieu de production du savoir. Et par transfert et appropriation, il n’y aurait plus qu'à appliquer. Un tel schéma convient bien aussi à toute bureaucratie.

Même s'il y a parfois des doutes, suscités par les ratés de ce processus présumé, le schéma structure profondément l'imaginaire et les comportements de nombreux acteurs de la recherche et de la «technostructure ». Prenons pour exemple un numéro de la revue des didacticiens des sciences consacré au thème «didactique des sciences et formation des enseignants » $\left(A S T E R \mathrm{n}^{\circ} 32,2001\right)$.

Voici les principales rubriques de la présentation générale du thème et des articles (Schneeberger \& Triquet, 2001):

- Premier pôle : la nature des savoirs didactiques utilisés en formation.

- La question de l'évolution des conceptions des enseignants,

- La question des savoirs didactiques à enseigner,

- La question de la transposition des résultats de recherche; transformation et distorsions; transposition des données de la recherche dans les pratiques «sans déformation abusive ».

- Deuxième pôle : les modèles de formation. Comment les modèles pédagogiques élaborés dans le contexte de la recherche peuvent-ils être adaptés à la formation des enseignants ?

- Troisième pôle : la pertinence professionnelle de la didactique des sciences.

- La question de la pertinence professionnelle de l'approche didactique. Participation de formateurs à la construction d'outils dans le cadre de recherches. 
- La question du réinvestissement dans les pratiques. Renouveler des pratiques par la recherche, résistances.

- Quatrième pôle : la relation entre le découpage disciplinaire et la formation.

- La question de la définition des contenus disciplinaires dans la formation et dans l'enseignement. Problème de la polyvalence des pratiques «transversales ».

- La question de l'interdisciplinarité. Collaboration avec des chercheurs d'autres disciplines.

Ce plan est tout à fait cohérent avec l'introduction, qui parle de «la » didactique qui a élaboré des outils théoriques et pratiques. De quelle «didactique » s'agit-il ?

On peut semble-t-il faire l'hypothèse que pour les auteurs de cette présentation, la didactique existe dans et par la recherche. Elle a produit des savoirs valides, des concepts, des faits, peutêtre des lois; elle se caractérise par une approche spécifique, l'«approche didactique » apparemment marquée par le constructivisme. Le but du numéro est d'explorer les possibilités et les difficultés de la recontextualisation dans la formation.

Quelles que soient les différences contextuelles ou essentielles, la didactique serait une ; c'est avant tout une discipline de recherche, conforme à une rationalité d'intelligibilité. Et lorsque les difficultés sont intrinsèques à la structure même de l'enseignement, il faudrait alors faire appel à d'autres disciplines de recherche. C'est une posture intellectuelle qui est ainsi adoptée, conduisant à ne rien imaginer d'autre que l'application du schéma transfert - application.

2. Pour réfléchir un peu plus avant, examinons rapidement des exemples de production au sein d'un laboratoire (le LIREST, devenu UMR.STEF) d'outils, et un exemple de reconfiguration du programme d'une discipline, pour en dégager à chaque fois les choix constructifs. 


\subsection{Production de guides pour la formation des enseignants}

Des membres du Laboratoire interdisciplinaire de recherche sur l'éducation scientifique et technologique (LIREST) ont publié au cours de la décennie 1990-2000 six guides pour la formation des enseignants dans la même collection d'un éditeur privé . D'autres guides ont été aussi publiés chez d'autres éditeurs. C'est dire que la question de la « mise à disposition » de savoirs pour la formation a été jugée fondamentale dans ce laboratoire de didactique des sciences et techniques (Goffard \& Weil-Barais, 2005).

On pourrait croire que provenant d'une même équipe, ces guides devraient se ressembler, non seulement dans la forme imposée par la collection, mais aussi dans les choix pédagogiques ou didactiques fondamentaux. Or, on ne peut ne peut qu'être frappé dès la lecture des sommaires par les différences des projets conçus et des choix effectués par les auteurs.

Dégageons rapidement ces choix constitutifs :

Construire des concepts en physique (Lemeignan \& Weil-Barais, 1993).

Le guide concerne quelques concepts de la mécanique, force, énergie et quantité de mouvement, dans l'enseignement secondaire supérieur scientifique. Il a pour base les recherches de ses auteurs sur l'enseignement et l'apprentissage de ces concepts. Il s'agit de transférer dans la pratique enseignante de classe les acquis de la recherche.

Observer pour comprendre les sciences de la vie et de la terre (Guichard, 1998).

Le domaine est celui de toute une discipline d'enseignement, à tous les niveaux du primaire et du secondaire. Le livre se fonde d'abord sur l'expérience et la documentation de l'auteur comme formateur. Mais ce dernier a approfondi par ailleurs certaines questions en recherche, il a participé à des formations innovantes.

Découverte de la matière et de la technique (Martinand et al., 1995). 
Le guide recouvre en neuf parties toutes les rubriques du programme de l'école maternelle et primaire concernant ces deux domaines, au moment de sa parution. Il s'efforce de présenter un ensemble systématique et souple pour mettre en œuvre le programme. La base est la double expérience de formation et de recherche des auteurs. Mais un important travail de cadrage théorique et de systématisation pratique a été effectué.

\section{Découverte du vivant et de la terre (Deunff et al, 1995).}

La visée est ici de faire réfléchir les maîtres de l'école primaire à la biologie (1 ${ }^{\text {ère }}$ partie), d'affirmer la spécificité et d'assurer la présence de l'enseignement de la biologie (« enseigner la biologie », $2^{\text {ème }}$ partie), et d'illustrer le propos didactique par des exemples d'activités scolaires à l'école primaire ( $3^{\text {ème }}$ partie) .

D Enseigner la technologie au collège (Lebeaume, Martinand et al, 1998).

Il s'agit ici d'un «compendium » pour les enseignants de technologie à l'école moyenne qui vise à leur donner une identité disciplinaire et en même temps à les munir des capacités pratiques pour enseigner le programme en vigueur. Les coordinateurs sont deux chercheurs en éducation technologique, qui ont aussi contribué à l'invention de la discipline, à sa mutation et au montage des programmes à l'époque de parution.

Dans tous les cas on s'aperçoit qu'il y a une relation à la recherche, ce qui est attendu lorsque de tels outils sont rédigés ou coordonnés par des chercheurs, mais avec des visées, des projets très différents, voire opposés. Cela nous impose deux questions : celle des statuts des produits de recherche d'une part, celle de la nature du travail de production de tels outils d'autre part, par rapport à la recherche, par rapport aux pratiques d'enseignement et de formation ensuite, par rapport aux normes qui régissent l'enseignement enfin (Martinand, 1994).

\subsection{Construction et reconstruction de curriculum}


À la même époque, la discipline technologie au collège (école moyenne), a été reconfigurée à l'occasion de la rénovation des programmes de collège. Ce qui s'est passé lors de ce travail auquel ont activement participé (comme coprésident du Groupe technique disciplinaire et membre) les coordinateurs du guide de technologie, est intéressant du point de vue de la recherche car les négociations, délibérations, décisions et reformulations ont dû être accompagnées d'un travail d'ordre théorique .

En effet, le texte de ces programmes est certes un compromis institutionnel et idéologique fixant des normes, mais c'est aussi le produit d'une élaboration conceptuelle explicite. Voici quelques aspects de cette élucidation, développée dans Martinand (2003).

D'abord, compte tenu de la charge idéologique des débats à propos de la culture technique, de sa place dans la culture générale, et donc des objections à une éducation technologique générale pour tous, il a fallu distinguer trois plans :

- des débats d'ordre politique qui portent sur les missions d'une éducation technologique, et sur l'éventualité de sa mise en œuvre dans la forme d'une discipline scolaire.

- des débats d'ordre éducatif qui portent sur la traduction de ces missions en finalités et moyens.

- des questions d'ordre scientifique qui portent sur la dynamique des activités conjointes des enseignants et des apprenants, et sur les fonctions que joue finalement cette éducation technologique dans la réalité de sa mise en œuvre, avec ses effets induits, souvent cachés.

On peut dire, selon un autre point de vue que les deux premiers plans correspondent aux curriculums prescrits (reconstitués à partir d'analyses des textes et discours de prescription, par référence à des curriculums possibles que le chercheur conçoit), et le troisième aux curriculums « produits », à court et à long terme, vus côté enseignant et côté apprenant. Ces 
curriculums «produits » sont inférés à partir de recueils de données observables, traces, enregistrements, entretiens, etc.

Au-delà, dans un registre spécifiquement didactique, les choix ont dû être rapportés à ce qu'on peut appeler des curriculums «potentiels» (ce que les enseignants et même leurs formateurs sont capables d'imaginer devoir et pouvoir réaliser en fonction de leurs habitudes, de leurs compétences, de leurs aspirations et de suggestions de curriculums possibles); ces curriculums potentiels sont des modélisations explicatives des écarts constatés entre curriculums prescrits et produits, ou des modélisations prédictives des écarts vraisemblables, ou des scénarios prospectifs « réalistes » en contexte; ce sont donc des repères pour passer à une généralisation qui ne soit pas seulement l'application d'un programme », comme s'il déterminait par lui-même les résultats attendus, mais une action prolongée de constitution des capacités d' « usage » du programme par les enseignants dans les classes, appelant évaluation et rectification.

Dans le cas de la technologie (collège), il a fallu expliciter et assumer le fait inhabituel que les missions éducatives attribuées à la discipline lors de sa constitution en 1984, et que la commission n'avait pas mandat de changer, conduisaient à ce que les normes du programme soient fondamentalement et majoritairement des normes de «processus » et non des normes de «produit» éducatif : dans ce type curriculaire, les prescriptions fixent avant tout les activités à ménager, les références à privilégier, les moyens à utiliser, et seulement secondairement quelques objectifs de «compétences notionnelles et instrumentales», conséquences attendues des activités de réalisation sur projet. L'ensemble prescriptif est radicalement différent de ceux du type curriculaire maintenant dominant, où les prescriptions directrices fixent des objectifs à atteindre par des activités de pur apprentissage. Seule une 
réflexion approfondie sur les types curriculaires et leurs implications permet d'éviter les incohérences.

Le type curriculaire de cette figure de la technologie est apparemment impensable pour des «théories » de la transposition didactique, très répandues en didactiques de science, ou des théories de la création scolaire, plus répandues en didactiques du français ou des sciences sociales, car elles impliquent toutes une assimilation entre activités scolaires et activités de pur apprentissage ; il est aussi impensable pour des conceptions plus communes en sciences de l'éducation, où le sens et le pilotage d'ensemble des activités (orientation - régulation évaluation) sont conceptualisés au moyen d'objectifs de comportements ou de compétences. Il y a ainsi remise en question d'idées répandues dans les «vulgates éducatives savantes» actuelles.

Dans cet esprit, et de manière détaillée, un certain nombre de problèmes ont dû être étudiés du point de vue didactique pour fixer contenus et démarches de façon plus pertinente, plus précise, en adéquation avec les missions et les contextes, et de façon plus cohérente par rapport aux dispositifs de mise en œuvre. Il s'agit d'achever la conception du curriculum prescrit pour permettre des réalisations de curriculums «produits» moins aléatoires ou déviantes, ce qui ne signifie en rien, au contraire, que tout doive être «normé » et imposé, car cela rendrait impossible toute activité conjointe de l'enseignant et des apprenants. Parmi ces problèmes majeurs, il faut citer :

- problème des choix de références extrascolaires des activités scolaires ;

- problème de l'invention d'activités nouvelles, selon des démarches nouvelles ;

- problème de la structuration scolaire, en une «technologie de l'information », des apprentissages pour une utilisation réfléchie des « nouvelles technologies » de traitement et de transfert de l'information ; 
- problème de l'unité de la discipline, à partir du moment où c'est une discipline portée par un corps social spécialisé, mais dans la diversité des références, des activités et de leur mode de pilotage ;

- problème de la progressivité des activités et des visées au long du cursus, selon des principes de progression multiples, ;

- problème de la flexibilité structurelle du curriculum pour qu'il puisse être adapté sans dénaturation à des publics et des contextes sociotechniques divers ;

- problème de l'« évaluabilité », en particulier pour les activités de réalisation sur projet ;

- problème de l'identité enfin ; identité interne de la discipline, ce qui a conduit à produire le guide évoqué précédemment, et identité externe, relationnelle, dans le système d'ensemble des disciplines et des actions éducatives formant le curriculum général du collège.

Le guide de formation (Lebeaume, Martinand et al, 1998) est la matérialisation de ces élaborations, mais le programme lui-même n'aurait pas pu être élaboré sans ce travail parallèle de critique et d'élucidation didactiques.

De nouveaux programmes appliqués à partir de 2007 dessinent une nouvelle figure de la discipline, transformée en discipline d'apprentissages de savoirs technologiques sur des thèmes techniques, sans d'ailleurs que cette réorientation ait fait l'objet d'une délibération approfondie par une commission légitime (voir Lebeaume dans cet ouvrage). Mais l'Education manuelle et technique, qui précédait la technologie (collège) avant 1985, présentait elle aussi une figure radicalement différente. C'est pourquoi l'éducation technologique, avec sa variabilité permanente et même des mutations fondamentales sur des périodes plus courtes que la durée d'une génération enseignante est un exemple de choix, un «modèle d'étude» pour mettre à l'épreuve les cadres d'analyse et de conception curriculaires, historiques, sociologiques ou didactiques. 
De fait, le curriculum prescrit construit entre 1995 et 2000 a eu une durée de vie d'une bonne dizaine d'année, pendant lesquelles il a bousculé les conceptions d'administrateurs et de formateurs, sans d'ailleurs les modifier profondément. Certaines questions de didacticiens des sciences ou de la technologie ont été caractéristiques de cet ébranlement: «mais est-ce que ce que dit le programme est bien ce que voulaient dire les auteurs ?»; parce que c'est contraire à la «forme scolaire», ou à la «forme disciplinaire», acceptées comme imposant des caractéristiques «génériques », « essentielles » et éternelles. Il est vrai qu'un tel curriculum soulève au sens fort des paradoxes qui invitent à élaborer des cadres de conception et d'analyse moins réducteurs.

Il faut souligner cependant que dans la littérature internationale de sciences de l'éducation de nombreux chercheurs ont proposé des cadres d'analyse ou de conception non orthodoxes, et surtout pluriels pour élucider des «problèmes curriculaires ». Ainsi le Britannique Alistair Ross (2000), en rapportant à trois types idéaux de curriculums (content oriented, objectives oriented, process oriented curricula) les délibérations et décisions de politique éducative en Angleterre pendant plus d'un siècle, a rendu intelligibles les relations complexes entre positionnements politiques partisans et orientations éducatives dans les débats sur l'école, avec les oppositions, les chevauchements et les évolutions (Ross, 2000).

3. Avec les deux exemples évoqués dans la deuxième partie, l'intention est de suggérer que si le schéma du transfert et de l'application correspond à certaines pratiques existantes, il est tout à fait insuffisant en général pour analyser, intervenir et élaborer en dehors du terrain de la recherche. C'est l'idée qui a présidé à une recherche coopérative de l'Institut national de recherche pédagogique (INRP, France).

La suggestion de départ, venant de la Direction de l'Enseignement Supérieur du Ministère, était d'étudier «l'utilisation des résultats de la recherche en éducation » (1996). Lors d'un 
premier appel d'offre INRP aux chercheurs en éducation, les propositions de recherche recueillies n'ont pas parues assez élaborées pour être retenues. Un second appel d'offre sur « le transfert des résultats de la recherche en éducation» (1998), n'a pas suscité de projets de travail plus pertinents, au-delà de la juxtaposition d'actions de formation particulières et partielles avec des évaluations sommaires. Un nouvel appel, après un séminaire d'un an de confrontation et de réflexion critiques sur l'idée de transfert a conduit à une recherche coopérative sur « la circulation des savoirs entre recherche, pratique et formation » (Derouet, 2002).

Dans les évolutions des pratiques d'enseignement et de formation, il n'est pas évident qu'en général, les résultats des sciences humaines et sociales jouent le rôle de moteur direct. Les changements résultent souvent de bricolages d'enseignants de terrain, pensés avec leurs concepts et leurs expériences, repris et relayés par différentes instances (centres de formation, mouvements pédagogiques, inspections et administrations, organisations syndicales). À l'échelle d'une génération, et du point de vue de l'histoire des idées, on peut cependant repérer l'impact de concepts et résultats produits dans le champ académique ; on peut même affirmer, à l'instar de Novoa (2001), que la plupart des idées qui s'imposent ont leur origine dans la recherche, mais lointaine. Si cela est vrai, il s'est produit entre temps une appropriation - transformation. Il n'y a pas eu de transfert immédiat ; il y a influence médiate. Pour rendre ces savoirs acceptables et efficaces dans la pratique enseignante ou formatrice, il ne s'agit pas tant de les « recontextualiser » pour ces pratiques ; cela conférerait à ces savoirs une valeur universelle en oubliant que les savoirs de recherche sont produits par ou plutôt au sein d'une pratique de recherche. Il faut donc plus qu'une recontextualisation, plus qu'une traduction ou une reformulation lorsqu'on change de pratique, il faut une reproblématisation et une réélaboration dans le cadre d'une autre pratique. C'est presque toujours l'hétérogénéité des pratiques qui est l'obstacle majeur, révélé lors de leur mise en relation (par exemple dans 
le cas des formations professionnelles alternées entre institution de formation et «terrain de stage» dans une organisation économique, administrative, ou culturelle); et pas l'absence de rapport entre « la » théorie et « la » pratique comme il est répété communément, ce qui n’a pas de sens clair, puisque qu'une « théorie » valide est toujours inscrite dans une pratique.

Lors d'un séminaire à l'INRP, le regretté Jean-Claude Forquin avait proposé de distinguer plusieurs « régimes de médiations» pour caractériser les voies d'influence des faits, propositions et questionnements issus des recherches :

- l'information-vulgarisation qui fabrique « l'air du temps »;

- l'appropriation de concepts et données par la technostructure pour conforter son autorité ;

- l'aide et le conseil pour surmonter des difficultés pratiques.

Mais il s'agit plutôt maintenant de penser ce qu'on pourrait appeler un régime d'expertise, où il faut recontextualiser, reformuler et surtout reproblématiser des savoirs scientifiques pour d'autres pratiques, en même temps qu'il faut aussi problématiser et donc reconceptualiser des expériences pratiques. Les circulations de savoirs et de compétences sont donc beaucoup plus complexes que ce que permettent d'en dire les schémas habituels. En des lieux, à des moments, avec des partenaires définis, ce régime d'expertise semble se nouer autour et par la problématisation lorsque des partenaires reconnaissent qu'ils n'ont pas de solution, même par transfert ou traduction.

Pour le chercheur, l'expertise est alors l'art de la reproblématisation, telle est la thèse qu'il est logique d'affirmer, en même temps qu'il doit accepter que pour les partenaires qui ne sont pas chercheurs, il s'agit d'un acte de co-problématisation, ce qui ne correspond d'ailleurs pas toujours à leurs demandes initiales. 
4. Dans un tel rapport partenarial, l'acte fondamental de problématisation à partir des enjeux abordés en commun, ne peut être qu'une co-construction, avec les partenaires. Pour que cette co-construction puisse être conduite, le chercheur doit accepter une certaine symétrie entre ce qu'apportent les partenaires et les chercheurs aussi bien dans leurs pratiques maîtrisées que dans leurs savoirs explicites voire implicites essentiellement inscrits dans ces pratiques.

Du point de vue de la pratique d'enseignement ou de formation, la pratique scientifique est jugée réductrice et parfois extravagante dans ses formulations, ses cadres conceptuels, ses intérêts. Réciproquement, du point de vue de la pratique de recherche, les pratiques enseignantes ou formatrices sont souvent jugées hétéroclites, irrationnelles. La sortie de la méfiance réciproque est rarement dans la résorption de ces «défauts », ou même la « traduction » proposée par Michel Callon (Lhomme \& Fleury, 1999), tant qu'il subsiste une négation des pratiques, des savoirs, des raisons, et des intérêts et des capacités chez l'autre. L'issue ne peut être que dans la recherche, conduite en collaboration, de réponses à un problème posé en commun.

Ce que nous devons donc penser et savoir ménager, c'est le partenariat, pas seulement en formation ou en intervention, mais aussi en recherche. Or les recherches «partenariales » en éducation sont essentiellement pensées comme recherches pour des « commanditaires ». Mais le commanditaire n'est pas exactement et immédiatement un «partenaire » pour la recherche, il s'agit de deux rôles différents, que les mêmes personnes ont du mal à équilibrer. Il apparaît alors que les chercheurs ont intérêt à varier les commanditaires (ministères, administrations centrales ou déconcentrées, conseils et services des collectivités territoriales, services de formation en entreprise, mais aussi associations, syndicats, commissions scolaires), pour ne pas être enfermés dans une «problématique » imposée, voire des « résultats attendus », et se trouver ainsi assignés à un rôle d'instrument au service d'un pouvoir. Tout autant, c'est la 
question du rôle des acteurs «de terrain » qui est posée : enseignants, personnels éducatifs, élèves, familles...qui ont aussi vocation à devenir des partenaires. Certes les recherches partenariales risquent de susciter des tensions et frictions institutionnelles et relationnelles : les partenaires nécessaires ne seront pas toujours disposés à « jouer le jeu » jusqu’à son terme, pas plus que les commanditaires initiaux et potentiels ne le sont.

L'éducation et la formation ont fait l'objet de réflexions et d'études nombreuses pour comprendre les spécificités des problèmes de l'action (par exemple Maggi et al, 2000) ou ceux de l'innovation (par exemple Cros, 1997). Ce ne sont pas les seuls domaines où des évolutions dans la direction de recherches partenariales s'imposent aux chercheurs comme aux commanditaires. L'expérience d'un institut français comme l'Institut national de la recherche agronomique, avec les réflexions, réorganisations, et échecs qui l'ont marquée est très suggestive (Sébillote, 2002): changement des thématiques, des modes de relations aux acteurs de terrain, quelquefois aussi instruits que les chercheurs, localisation et évaluation des chercheurs, etc. Les difficultés pointées sont de nature institutionnelle avec des incohérences entre les décisions, les demandes, la «gouvernance», l'évaluation, et aussi de nature «culturelle» chez les commanditaires et les partenaires. Mais la «culture épistémologique » des chercheurs est aussi en cause, l'exigence de scientificité, empirique et théorique, pouvant se doubler d'un certain mépris pour les acteurs («sujets ») et les partenaires (Darré, 1999).

Du côté des chercheurs, la recherche partenariale est un facteur de déstabilisation épistémologique. Pour ceux qui ont une conception de la recherche scientifique comme pure production de connaissances validées, la recherche partenariale exige en effet de passer de recherches descriptives et explicatives, à des recherches prédictives et plus encore prospectives, intégrant dans les hypothèses les conséquences et les implications des actions que la recherche peut contribuer, et contribue partiellement à engendrer. En d'autres termes, il 
s'agit pour certaines des recherches de passer d'une conception rétrospective à une conception proactive, qui puisse être partagée avec les partenaires et commanditaires. Ce mouvement, largement engagé aujourd'hui, selon des modalités plus ou moins imposées et acceptables qui font débats et affrontements, semble irréversible : la recherche et les chercheurs sont «en société », ils ne sont plus, s'ils l'ont jamais été «hors société ». Ils ne peuvent pas mettre à l'écart l'expertise comme fonction normale des chercheurs.

Le coeur de l'expertise, ce n'est pas la proposition de solutions, c'est d'abord la problématisation en situation avec des partenaires. À première vue, il s'agit d'une mise en cause des chercheurs, de leur savoir, et de la recherche. Mais s'il existe des «professionnels » de la problématisation, c'est bien dans la recherche qu'on peut les trouver. Si leur rôle de détenteur de vérités prouvées et surtout de solutions validées est dorénavant contesté, leur parole sur ce plan étant comparable à celle d'un citoyen quelconque proposant et argumentant son opinion, c'est parmi eux que sont cherchés les spécialistes de l'aide à la problématisation, c'est à eux que sont adressés les demandes et les appels d'offre de nombreux commanditaires potentiels. C'est une responsabilité universitaire que d'orienter le doctorat et le moment postdoctoral vers la constitution de telles capacités.

\section{Références :}


Burguière, E, Martinand, J-L (2002). Entretien. Recherche et Formation, $\mathrm{n}^{\circ}$ 40.Paris : INRP.

Cros, F. (1997). L’innovation en éducation et en formation. Revue française de pédagogie, $118, \mathrm{p} 127-156$.

Darré, J.-P. (1999). La production de connaissance pour l'action; arguments contre le racisme de l'intelligence. Paris : Éd. MSH \& INRA.

Derouet, J-L (coord, 2002). Du transfert à la circulation des savoirs et à la reproblématisation.

De la circulation des savoirs à la constitution d'un forum hybride et de pôles de compétences :

un itinéraire de recherche. Recherches et Formations, $\mathrm{n}^{\circ}$ 40. Paris : INRP.

Deunff, J (coord., 1995). Découverte du vivant et de la terre . Paris : Hachette.

Goffard, M. \& Weil-Barais, A. (dir., 2005). Enseigner et apprendre les sciences. Recherches et pratiques. Paris : A. Colin.

Guichard, J \& Deunff, J (coord., 1998), Observer pour comprendre les sciences de la vie et de la terre. Paris : Hachette.

Lebeaume, J \& Martinand, J-L (coord.,1998). Enseigner la technologie au collège. Paris : Hachette.

Lemeignan, G \& Weil-Barais, A (1993). Construire des concepts en physique. Paris : Hachette.

Lhomme, R.\& Fleury, J. (1999). Entretien avec Michel Callon - pour une sociologie de la traduction en innovation. Recherche et Formation, 31, p 113-125.

Maggi, B. (dir.) (2000). Manières de penser, manières d'agir en éducation et en formation. Paris : P.U.F.

Martinand, J.-L. (1994). La didactique des sciences et de la technologie et la formation des enseignants. ASTER, 19, p 61-75.

Martinand, J-L (coord., 1995). Découverte de la matière et de la technique. Paris : Hachette. 
Martinand, J.-L. (2003). L'éducation technologique à l'école moyenne en France : problèmes de didactique curriculaire. La revue canadienne de l'enseignement des sciences des mathématiques et des technologies, 3 :1,p 101-116.

Ross, A (2000). Curriculum. Construction and Critique.London : Routledge-Farmer.

Schneeberger, P, ;Triquet, E, (2001). Didactique et formation des enseignants. Des recherches en didactiques des sciences à la formation des enseignants : quels liens, quelles interactions ? ASTER, 32, 3-13.

Sébillotte, M. (2002). Logiques de l'agir et construction des objets de connaissance. L'invention de nouveaux dispositifs de recherche, p 93-115, in T. Gaudin \& A. Hatchuel (coord.) : Les nouvelles raisons du savoir. La Tour d'Aigues : éditions de l'aube.

Mots pour index : applications, conception de curriculum, didactique, expertise, formation des enseignants, problématisation, production didactique, recherche, transfert. 